\title{
Propagation of polarized mechanical waves in an anisotropic medium
}

\author{
Sergej Faletič ${ }^{1}$, Mojca Čepič $\check{c}^{2,3}$ \\ ${ }^{1}$ University of Ljubljana, Faculty of Mathematics and Physics, Jadranska ulica 19, \\ 1000 Ljubljana, Slovenia \\ ${ }^{2}$ University of Ljubljana, Faculty of Education, Kardeljeva ploščad 16, 1000 \\ Ljubljana, Slovenia \\ ${ }^{3}$ Institute Jožef Stefan, Jamova 39, 1000 Ljubljana, Slovenia \\ E-mail: sergej.faletic@fmf.uni-lj.si
}

\begin{abstract}
.
During the propagation in an anisotropic medium, linearly polarized light in general becomes elliptically polarized. One can detect the polarization state of the transmitted light, but not the evolution of the polarization state during the propagation inside the material. We present a mechanical model where this evolution can be observed. The anisotropic medium is modelled by a series of coupled anisotropic oscillators. An oscillator consists of a bead hanging on an elastic string, and is coupled to neighbouring oscillators with stronger springs. When the system is excited in the plane perpendicular to the springs, the trajectories of the oscillating beads indicate the polarization state of the excitation at their respective positions. We analyze the model analytically, numerically and experimentally.
\end{abstract}

\section{Introduction}

Understanding of polarized light propagation inside an anisotropic medium is difficult even for good physics students [1]. It is common practice in teaching physics to discuss waves and polarization first on mechanical systems, usually strings, before extending the concepts to electromagnetic waves. Direct observations of mechanical systems provide an experiential basis for the construction of mental models. It is also in line with a constructivistic approach to physics teaching, which emphasizes progress from the known to the unknown. However, an extensive literature search yielded no reports on real-life mechanical wave propagation in anisotropic media. Providing a mechanical system, which exhibits the discussed behaviour, appears to be a missing link in the teaching chain. The experiment described herein can be used as an observation experiment for building new knowledge or as a testing experiment for testing the result of the mathematical model. In both cases it offers support for active learning. But, it can also be used as a demonstration experiment in a traditional setting.

An anisotropic medium in general does not preserve the polarization state of a propagating wave. Inside an anisotropic material, a wave propagates as two waves 
with different phase velocities and mutually perpendicular polarizations. The two polarization directions we call vibration directions, in accordance with [2]. Therefore, the phase difference between the two waves changes with depth, so the polarization state of the actual (superposed) wave also changes with depth. A linearly polarized wave remains linearly polarized inside the medium, only if its polarization direction corresponds to one of the vibration directions.

A research study [1] shows that novices and sometimes even experts have erroneous or incomplete ideas about the evolution of a polarization state inside the medium. To help the visualization of the phenomenon several freely accessible animations and simulations (e.g. EMANIM [3]) have been developed. However, such information communication technology (ICT) tools are constructed from mathematical models, which are idealized, and can reinforce the impression that physics is based on mathematical models rather than reality. It is therefore relevant to develop a reallife tool showing that the results of simulations match well with reality. In this context, simulations can provide a clearer view of the phenomenon, which can serve to show the students what to focus their attentions on when observing the real-life phenomenon.

In literature, several reports discuss the propagation of electromagnetic waves in anisotropic media, mostly light (see e.g. [4-7]), but also microwaves [8]. Reports that address mechanical systems, can be sorted into three groups. The first group does not discuss wave propagation, but focuses on two-dimensional oscillations instead. In [9] two perpendicularly intersecting wires, each connected to a pendulum and oscillating with its frequency, are used to demonstrate the elliptical oscillation of their intersection. The frequencies of the pendula are the same, but the phase difference can be adjusted. In [10] a bead is connected to a frame by two conducting wires, one on each side. One wire is placed in a vertical and the other in a horizontal magnetic field. The wires are connected to function generators, which provide alternate currents to the wires with adjustable phase difference between them. The trajectory of the oscillating bead can consequently be linear, elliptical or circular, depending on the phase difference. A popular method for showing the elliptical oscillation is to use an oscilloscope in $x-y$ setting with adjustable phase difference between the channels (see for example [11]). The experiments described in these reports demonstrate the elliptical polarization and the role of the phase difference, however, they only discuss the motion of one point oscillations - they do not address travelling waves.

The second group focuses on tools for the visualization of the propagation of an elliptically polarized wave. In [12] an apparatus is presented which consists of a chain and an upwards oriented rod fixed on the chain, which can move forwards as the chain is pulled. Small screens with slits are placed at different positions along the path of the rod and at different polarization angles. As the rod moves, the screens with slits rotate so that the rod passes through each of them. The rod represents the phase of the wave and its motion represents the propagation of the phase. The slits in the screens represent the polarization direction of different segments of the wave at a particular time - the wave form. In [13] a cardboard 3D model is presented. It consists of two sinusoidal shapes 
cut out of cardboard, which are placed perpendicularly to each other and can be slid back and forth to adjust their phase difference. The elliptical polarization is visualized at any distance by the vector sum of the two components represented by the sinusoidal shapes. The sum of the two components is not produced by the apparatus, it has to be visualized by the observer. Both reports describe tools to support visualization. Nowadays, the same can be achieved with a computer simulation such as EMANIM [3]. Other simulations that address the propagation of an elliptically polarized wave include the PhET simulation Wave interference [14] where the motion of air molecules in a sound wave is elliptical, and a Gizmos simulation Waves [15] of a wave on a string which can be driven longitudinally and transversally at the same time, producing an elliptical motion of its segments. However, in both these simulations, the elliptical motion is a combination of longitudinal and transversal components, and they address propagation in an isotropic medium, while EMANIM visualizes purely transversal two dimensional waves with the option of an anisotropic medium. In all these tools the direction of the visualized displacement or electric field vector at any moment and at any position is predetermined by the design of the visualization tool.

In the third group reports discuss systems of coupled oscillators, like the one described in this report. These systems in their continuous limit are described by the Klein-Gordon equation, and are therefore sometimes called Klein-Gordon strings [16-19]. The reports discuss the use of the systems as classical analogies or visualization tools for quantum phenomena related to the Klein-Gordon equation. For this purpose, the displacement is limited to one plane, parallel to one vibration direction, and it remains linear troughout the medium.

The experiment presented in this contribution adds to the previous research in the following ways. First, the Klein-Gordon string is excited linearly at an angle with regard to the vibration directions. This excites two mutually perpendicularly polarized waves with different propagation velocities. Second, the propagation of the wave and its polarization state can be visually followed throughout the medium, so not only the elliptical polarization at any given point inside the medium can be observed, but also the propagation of the wave itself, i.e. the visual following of the motion of crests and troughs that is often associated with the meaning of the phrase 'wave propagation'. Third, the phase difference and the change of polarization state occur due to easily identifiable mechanisms: the anisotropic properties of the oscillators, discussed in greater detail later on, and, of course, the coupling between the oscillators, all of which are described by Newtonian mechanics. Fourth, the system is designed to minimize longitudinal polarization, which allows for a closer comparison with electromagnetic waves.

In Section 2, the theoretical description of the system is provided and the velocities of the two mutually perpendicularly polarized waves are derived. Section 3 describes the realization of the Klein-Gordon string. The evolution of the polarization state is recorded with a camera and the results are compared to the theoretical predictions. The final section summarizes the conclusions and discusses the potential use of the experiment in education. 


\section{Theoretical description}

The Klein-Gordon string, presented in this paper, consists of a series of coupled oscillators (Fig. 1). An oscillator consists of a bead attached to an elastic string, called z-spring. It behaves as a spring oscillator in the vertical direction and approximately as a pendulum in the horizontal direction. The longitudinal coupling between the oscillators is provided by springs connecting the beads, called x-springs. The coupling and the excitation frequency are chosen so that the energy transfer between the oscillators is much faster than one oscillation period. The beads and the couplings between them form a springs-and-beads type of string - a medium, along which horizontally polarized waves propagate with a different velocity than vertically polarized waves.

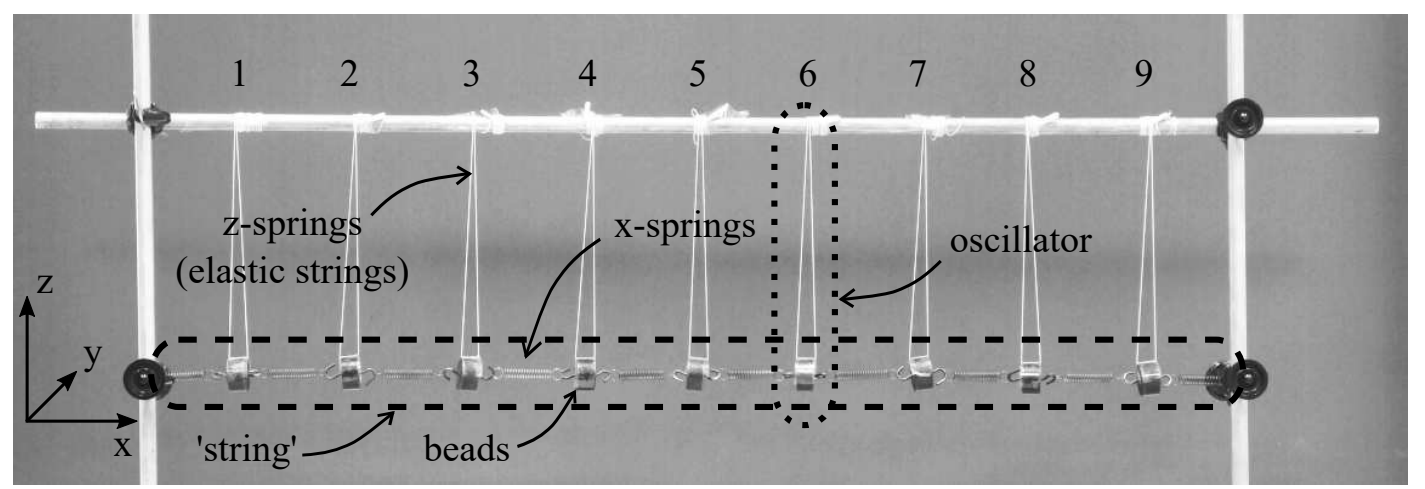

Figure 1. A picture of the apparatus. The z-springs are elastic strings and with the attached beads form spring oscillators in the vertical direction $z$, or pendula in the horizontal direction $y$. The coupling between these oscillators is provided by the $\mathrm{x}$-springs, which are metal springs. The $\mathrm{x}$-springs and the beads are considered a springs-and-beads type of string. The oscillators are numbered consistently with Fig. 2.

To proceed with a theoretical description of the dynamics, the coordinate system is set in the following way: The direction of the wave propagation is denoted $x$, the vertical direction $z$ and the horizontal $y$. The directions $x, y$ and $z$ are mutually orthogonal. The forces acting on each bead determine its acceleration. The vertical component of the acceleration is determined by

$$
T \Delta x \frac{\Delta^{2} z}{\Delta x^{2}}-b_{z} z=m \frac{\partial^{2} z}{\partial t^{2}}
$$

where $T$ is the tension of the string, $\Delta x$ is the distance between neighbouring beads, $b_{z}$ is the coefficient of the z-springs, $m$ is the mass of one bead, $z$ is the vertical displacement of the bead, $x$ is the coordinate along the medium, and $t$ is time. $\Delta^{2} z=\left(z_{i+1}-z_{i}\right)-\left(z_{i}-z_{i-1}\right)$, if $z_{i}$ is the displacement of the observed bead, and $z_{i+1}$ and $z_{i-1}$ are displacements of the neighbouring beads. The first term in (1) describes the forces of the $\mathrm{x}$-springs on one bead, and the second term describes the forces of the $\mathrm{z}$ springs on the same bead. Because the experimental system is discrete, the equations are 
written in the form of differences. This form is transformed into a differential equation

$$
\frac{T}{\mu} \frac{\partial^{2} z}{\partial x^{2}}-\frac{\beta_{z}}{\mu} z=\frac{\partial^{2} z}{\partial t^{2}}
$$

by approximating $\Delta x$ to zero, while keeping $\beta_{z}=b_{z} / \Delta x$ and $\mu=m / \Delta x$ constant. In practice this means ever lighter beads on ever softer z-springs with ever more of them per unit length.

The Ansatz for the solution of (2) is

$$
z=z_{0} \cos (k x-\omega t+\delta)
$$

where $z_{0}$ is the amplitude of the displacement, $k$ is the wave vector, $\omega$ is the angular frequency of the excitation, and $\delta$ an arbitrary phase. The phase velocity of vertically polarized waves is then

$$
c^{2}=c_{0}^{2} \frac{1}{1-\left(\frac{\omega_{b}}{\omega}\right)^{2}},
$$

or with $c=\omega / k(\omega)$

$$
k(\omega)=\frac{\omega}{c_{0}}\left(1-\left(\frac{\omega_{b}}{\omega}\right)^{2}\right)^{1 / 2},
$$

where

$$
c_{0}^{2}=\frac{T}{\mu}
$$

is the phase velocity on the string alone, without z-springs, and

$$
\omega_{b}^{2}=\frac{\beta_{z}}{\mu}
$$

In the discrete description (1), this is the natural frequency of one oscillator $\omega_{b}=$ $\sqrt{b_{z} / m}$.

For the horizontal direction, $y$, the situation is different. When the bead is displaced from the equilibrium position it behaves like a pendulum, but an additional force due to the stretching of the z-spring also influences its motion. The total force caused by the $\mathrm{z}$-spring is

$$
F_{y}=b_{z}\left(l\left(1+\left(\frac{y}{l}\right)^{2}\right)^{1 / 2}-L\right) \frac{y}{l} .
$$

Here, $y$ is the horizontal displacement, $l$ is the length of the z-springs, when the string is at rest, and $L$ is their own rest length when disconnected. For small $y / l$, the expression is expanded in Taylor series to the second order in $y / l$. Taking into account the equilibrium condition $(l-L) b_{z}=m g$, (8) becomes

$$
F_{y}=m g \frac{y}{l}+b_{z} l\left(\frac{y}{l}\right)^{3} \text {. }
$$


Here the first term represents a pendulum of length $l$ on a rigid string, and the second term appears due to the stretch of the z-springs. For

$$
\begin{aligned}
& \left(\frac{y}{l}\right)^{3}<1 \quad \text { and } \\
& \frac{y}{l} \quad<2(1-L / l),
\end{aligned}
$$

the second term will always be much smaller than the first. The first criterion is already fulfilled if Taylor expansion to the second order is adequate. The second criterion sets the upper limit to the $y$ displacement of the bead. In this approximation, the system is linear. The equation of motion in finite difference form is then

$$
T \Delta x \frac{\Delta^{2} y}{\Delta x^{2}}-m \frac{g}{l} y=m \frac{\partial^{2} y}{\partial t^{2}}
$$

and the corresponding differential equation is

$$
\frac{T}{\mu} \frac{\partial^{2} y}{\partial x^{2}}-\frac{g}{l} y=\frac{\partial^{2} y}{\partial t^{2}}
$$

This equation has the same form as Eq. (2), with one difference: The second term has a different constant, which corresponds to the natural frequency of a pendulum

$$
\omega_{g}^{2}=\frac{g}{l} \quad .
$$

The expression $\omega_{g}$ replaces $\omega_{b}$ in (4) and (5), and the phase velocity of horizontally polarized waves is

$$
c^{2}=c_{0}{ }^{2} \frac{1}{1-\left(\frac{\omega_{g}}{\omega}\right)^{2}} .
$$

Comparison of phase velocities given by (4) and (15) shows that the phase velocities of vertically and horizontally polarized waves are different. The medium is, therefore, effectively anisotropic. Such a medium allows for reproduction of some effects characteristic for propagation of light in anisotropic materials, namely the evolution from linear to elliptical polarization of the wave and vice versa.

\section{The experiment}

The 'string' medium was composed of nine beads of $m=(50 \pm 2) \mathrm{g}$, connected with $\mathrm{x}$ springs of $1.0 \mathrm{~cm}$ rest length, and a coefficient of $(0.525 \pm 0.014) \mathrm{N} / \mathrm{cm}$. The beads were $\Delta x=(9.5 \pm 0.5) \mathrm{cm}$ apart, and the lengths of the stretched $\mathrm{x}$-springs were $(4.5 \pm 0.2) \mathrm{cm}$, under the tension of $T=(1.84 \pm 0.15) \mathrm{N}$. The beads were connected to a support frame with elastic strings from common elastic bands with spring coefficient $b_{z}=(8.33 \pm 1.5) \mathrm{N} / \mathrm{m}$, sold as material for sewing. The elastic strings had the role of zsprings. Their rest length was $L=(11.6 \pm 0.5) \mathrm{cm}$ and their loaded length in equilibrium position was $l=(17 \pm 0.5) \mathrm{cm}$. These parameters yield $2(1-L / l) \approx 0.7$, which satisfies criterion (11). From these data and Eqns. (7) and (14) follows $\omega_{b}=(12.9 \pm 1.3) \mathrm{s}^{-1}$ and $\omega_{g}=(7.60 \pm 0.12) \mathrm{s}^{-1}$, respectively. 
To follow the evolution of the polarization state inside the medium, we excited the first bead at a $45^{\circ}$ angle in the $\{z, y\}$ plane with a pulse resembling a half-sine with the amplitude $y / l \approx 0.3$ satisfying criterion (10). The propagation of the excitation was recorded with a video camera at 120 frames per second. The trajectories of each bead were extracted with a video analysis software [20], and are shown in Fig. 2(a). The motion of the beads was filmed from a position close to the $x$ axis. The position of each bead was then manually marked in the software frame by frame. Since the displacement of the beads is comparable to the size of the beads (the displacement should be kept small to maintain the approximation used to arrive a the linear equation (12)), small deviations may occur when selecting the point to mark. This point was an identifiable point on the bead (the connection to the z-spring), but due to small rotations of the bead and the finite resolution of the video, this point may not perfectly represent the motion of the centre of mass of the bead or the motion of the point particle-bead in the model. All these imperfections add some noise to the measured trajectories of the beads.

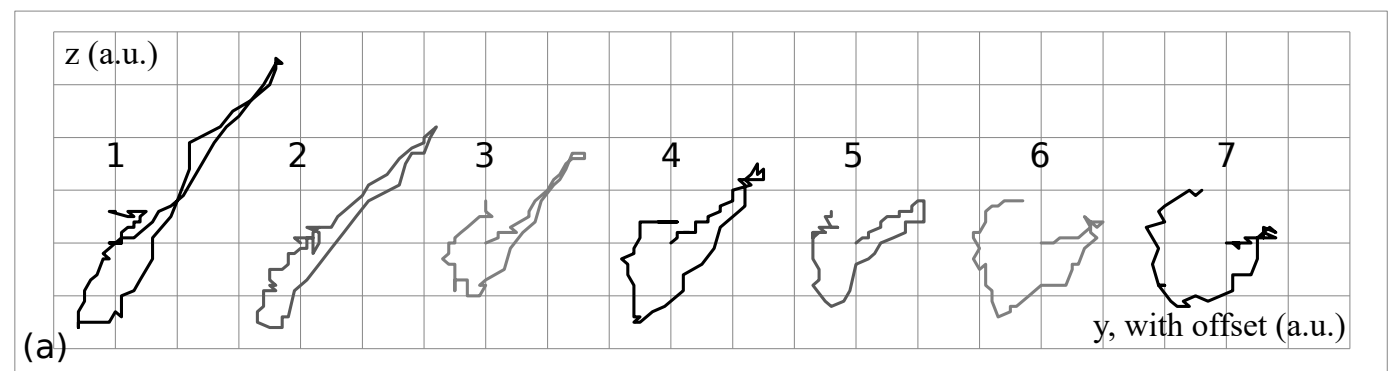

(a)

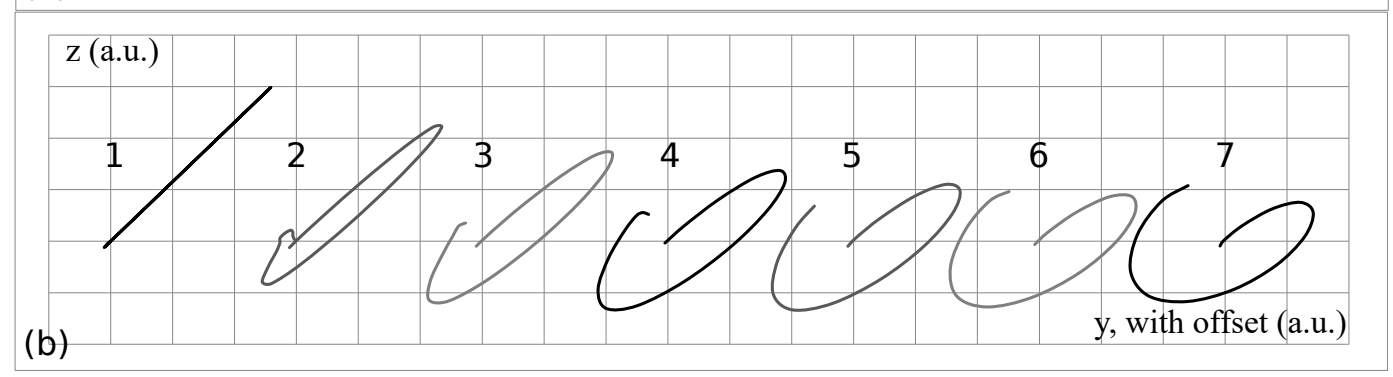

Figure 2. Trajectories of the beads for the incident wave. Experimental data (a) and theoretical calculation (b). The rest position of each bead is offset from the previous to provide a clearer view of its trajectory. Bead numbers correspond to the numbering in Fig. 1. The comparison between (a) and (b) clearly shows that the experimental data and theoretical predictions are in good qualitative agreement.

The trajectories in Fig. 2(a) clearly demonstrate the evolution from linear polarization state of the first bead towards a circular polarization state. Around the $7^{\text {th }}$ bead the trajectory already closely resembles a circular polarization state. The trajectories of beads 8 and 9 are excluded from the analysis, because the influence of the reflected wave on their trajectories is significant, and makes the extraction of the trajectories due to the incident wave impossible.

The theoretical prediction from model (3) is straightforward for waves with a well 
defined frequency only. However, the excitation in the experiment was a time-limited pulse of a half-sine form, therefore its decomposition into sinusoidal components by Fourier analysis was applied. The pulse is written as a product of a sine with frequency $\omega_{0}=2 \pi / t_{0}$ and a rectangular function to describe the duration of the pulse

$$
\begin{aligned}
& z(0, t)=z_{0} \sin \left(\omega_{0} t\right) \operatorname{rect}\left(\frac{t-t_{0} / 4}{t_{0} / 2}\right) \\
& y(0, t)=y_{0} \sin \left(\omega_{0} t\right) \operatorname{rect}\left(\frac{t-t_{0} / 4}{t_{0} / 2}\right)
\end{aligned}
$$

where

$$
\operatorname{rect}(t)=\left\{\begin{array}{lll}
1 & ; \quad & -1 / 2 \leq t \leq 1 / 2 \\
0 & ; & \text { elsewhere }
\end{array} .\right.
$$

The driving frequency $\omega_{0}=(11.6 \pm 0.6) \mathrm{s}^{-1}$ was determined with the video analysis. With the well known transforms

$$
\begin{aligned}
& \mathrm{FT}\left(\sin \left(\omega_{0} t\right)\right)=-i \pi\left(\delta\left(\omega-\omega_{0}\right)-\delta\left(\omega+\omega_{0}\right)\right) \\
& \mathrm{FT}(\operatorname{rect}(t / T))=T \operatorname{sinc}(\omega T / 2) \\
& \mathrm{FT}(f(t-T))=F(\omega) \exp (-\mathrm{i} \omega T)
\end{aligned}
$$

where $\mathrm{FT}(\ldots)$ denotes the Fourier transform, $\operatorname{sinc}(x)=\sin (x) / x$, and $F(\omega)$ is the Fourier transform of $f(t)$

$$
F(\omega)=F T(f(t))=\int_{-\infty}^{\infty} f(t) \exp (\mathrm{i} \omega t) d t \quad,
$$

the Fourier transforms of $z(0, t)$ and $y(0, t)$ are

$$
\begin{aligned}
& F_{z}(\omega)=\operatorname{FT}(z(0, t))=z_{0} \frac{2}{\omega_{0}} \frac{\cos \left(\pi / 2 \omega / \omega_{0}\right)}{1-\left(\omega / \omega_{0}\right)^{2}} \exp \left(\mathrm{i} \pi / 2 \omega / \omega_{0}\right) \\
& F_{y}(\omega)=\operatorname{FT}(y(0, t))=y_{0} \frac{2}{\omega_{0}} \frac{\cos \left(\pi / 2 \omega / \omega_{0}\right)}{1-\left(\omega / \omega_{0}\right)^{2}} \exp \left(\mathrm{i} \pi / 2 \omega / \omega_{0}\right)
\end{aligned}
$$

The signal at an arbitrary position and time is

$$
\begin{aligned}
& z(x, t)=\frac{1}{2 \pi} \int_{-\infty}^{\infty} F_{z}(\omega) \exp \left[\mathrm{i}\left(k_{z}(\omega) x-\omega t\right)\right] d \omega \\
& y(x, t)=\frac{1}{2 \pi} \int_{-\infty}^{\infty} F_{y}(\omega) \exp \left[\mathrm{i}\left(k_{y}(\omega) x-\omega t\right)\right] d \omega .
\end{aligned}
$$

The integrals were calculated numerically with $N=128$ components in the spectrum and $\Delta \omega=0.44 \mathrm{~s}^{-1}$ the difference in frequency between those components. A good qualitative correspondence between the measured data and the theoretical prediction was achieved already if the spectrum was truncated at the second minimum, which occurs at $\omega=4 \omega_{0}$. To calculate both wavevectors $k_{z}(n \Delta \omega)$ and $k_{y}(n \Delta \omega)$ we insert $\omega_{b}$ and $\omega_{g}$ into (5). Trajectories, calculated from (21) are shown in Fig. 2(b).

The experimental (Fig. 2(a)) and the theoretical (Fig. 2(b)) results are clearly consistent. The theoretical results from (21) based on the model described by the 
two wave equations (2) and (13) efficiently predict the evolution of the polarization state. This confirms that the described medium is in fact an anisotropic medium for mechanical waves, which can be used to study the evolution of polarization states in anisotropic media. The mechanical nature of the apparatus allows for a clear insight into the evolution of a polarization state inside the medium and the mechanisms giving rise to it. In addition, the theoretical description involves only mathematics at the undergraduate level. It also provides an example of efficient use of Fourier analysis beyond data analysis.

\section{Discussion and conclusions}

This study was motivated by frequent observations that in-service teachers, preservice teachers, physics students and to a degree even physics professionals have problems understanding how a polarization state of an electromagnetic wave evolves inside an anisotropic material. Therefore, to support the development of conceptual understanding, a mechanical medium using simple components such as beads, springs and strings was constructed. The Klein-Gordon string presented in this paper is an anisotropic medium for mechanical waves, which clearly exhibit an evolution of the polarization state similar to electromagnetic waves in anisotropic materials. The system minimizes the longitudinal motion of the beads and emphasizes their transversal motion, which allows for a better comparison with electromagnetic waves.

There are several similarities between the propagation of mechanical waves along the Klein-Gordon string in this report and electromagnetic waves in anisotropic materials. In the presented apparatus, vertically polarized waves propagate with different phase velocity than horizontally polarized waves. This phenomenon is similar to the propagation of electromagnetic waves in anisotropic materials, where waves split into two mutually perpendicularly polarized waves with two different phase velocities. In electromagnetic waves, the polarization state changes with position inside the material, and is characterized by the characteristic ellipse. In the presented apparatus, the polarization state of each bead is characterized by the trajectory of the bead. Therefore, the trajectories of the beads can be considered as a model for the polarization states of the electromagnetic wave. This apparatus allows for direct observation of the trajectories of each bead and thus the polarization state at different positions inside the material, which is impossible for electromagnetic waves.

The theoretical model is in good qualitative agreement with experiments. Despite similar results, the mechanisms for the mechanical and electromagnetic waves are different. However, in the Klein-Gordon string, the mechanisms are easily identifiable and are easily related to the difference in natural frequencies of the oscillators in two perpendicular directions. The apparatus has some limitations. The motion of the last two beads of the Klein-Gordon string was excluded from the analysis due to the reflection of the wave from the end of the string. The reflected wave can be modelled and similarities are still identifiable, however, due to damping, which over longer distances 
cannot be avoided, and due to dispersion and the consequent widening of the pulse and lowering of the amplitude, the wave shapes become more difficult to discern, and comparison with the model becomes more difficult. A longer string could be constructed, but it would run the risk of becoming impractical for use in the classroom due to its size. Smaller beads could facilitate the visual tracking with the video analysis software and reduce the noise of the measured trajectories. However, the apparatus is primarily intended for providing a visual impression of the wave propagation in an anisotropic medium. Therefore, it has to be large enough for the audience to clearly see its construction and the motion of the beads. The change in polarization can be seen without measurements (see pictures on the web page [21]). The video analysis is more appropriate as an extension for more motivated students.

The apparatus can be useful as an experimental support for anyone studying polarization, dispersion and wave propagation, but it might be especially useful for educators. The simple construction and purely Newtonian mechanisms offer the opportunity for the students to do any or all of the following: observe the phenomenon of polarization change. See the construction and recognize the asymmetry of the oscillators, and identify the different modes of oscillation. Realize that the modes in general do not have the same natural frequency, which is crucial. Form hypotheses to explain the phenomenon, of which at least some will likely include the anisotropy of the oscillators. Suggest testing experiments, which may include changing the parameters of the apparatus. A short, large and modular construction can make it possible to actually do that, like changing the properties of the z-springs. Using a large number of beads would make this very time consuming. More advanced students can proceed to building a theoretical model and to the application of the Fourier transform to analyse the propagation of a pulse.

Overall, the apparatus provides a means to observe the phenomenon of polarization change in a real, mechanical medium, and the opportunity to build upon this observation towards any related goal, along with the possibility to compare the observations with the theoretical results. However, we believe that its mechanical construction can be most benefitted from when addressing the role of natural frequencies of the oscillators in the emergence of the phenomenon.

\section{References}

[1] M. Pečar. Konoskopija in poučevanje anizotropnih optičnih lastnosti snovi/Conoscopy and teaching anisotropic optical properties of matter [in Slovenian]. PhD thesis, University of Ljubljana, Faculty of mathematics and physics, Slovenia, 2016.

[2] Maja Pečar and Mojca Čepič. Conoscopic figure: a complex consequence of a not so simple phenomenon. Eur. J. Phys., 36(3), 2015.

[3] EMANIM. Animations of electromagnetic waves, 2014. http://emanim.szialab.org/ [Online; accessed 29-January-2018].

[4] Terrill W. Mayes. Polar intensity profile of elliptically polarized light. Am. J. Phys., 44(11), 1976.

[5] A. J. Cox. Quantitative analysis of elliptically polarized light. Am. J. Phys., 46(3), 1798. 
[6] P. C. B. Fernando and S. Samarasinghe. A technique for experimentally observing the polarization ellipse. Am. J. Phys., 64(5), 1996.

[7] Augusto Beléndez, Elena Fernández, Jorge Francés, and Cristian Neipp. Birefringence of cellotape: Jones representation and experimental analysis. Eur. J. Phys., 31(3), 2010.

[8] Saša Ziherl, Jurij Bajc, Bernarda Urankar, and Mojca Čepič. Anisotropy of wood in the microwave region. Eur. J. Phys., 31(3), 2010.

[9] B. H. Morgan. Polarization effects with pendulums. Phys. Teach., 20, 1982.

[10] R. E. Stevens. A simulation of optical polarization. Phys. Teach., 38, 2000.

[11] L. E. McNeil and D. E. Holmgren. An elliptical polarization machine. Phys. Teach., 64, 1996.

[12] W. Herreman and H. Notebaert. Circularly polarized waves: A mechanical demonstration. Am. J. Phys., 51, 1983.

[13] E. Torigoe. Representing circular polarization with a box of cereal. Phys. Teach., 50, 2012.

[14] PhET. Wave interference, 2018. https://phet.colorado.edu/en/simulation/legacy/ wave-interference [Online; accessed 19-January-2018].

[15] Gizmos. Waves, 2018. https://www.explorelearning.com/index.cfm?method=cResource. dspDetail\&resourceID=1053 [Online; accessed 23-January-2018].

[16] P. Gravel and C. Gauthier. Classical applications of the klein-gordon equation. Am. J. Phys., 79, 2011.

[17] A. Mouchet. Interaction with a field: a simple integrable model with backreaction. Eur. J. Phys., 29, 2008.

[18] E. Bertozzi. Hunting the ghosts of a 'strictly quantum field': the klein-gordon equation. Eur. J. Phys., 31, 2010.

[19] S. Faletič. A mechanical wave system to show waveforms similar to quantum mechanical wavefunctions in a potential. Eur. J. Phys., 36, 2015.

[20] Vernier. Logger pro homepage, 2017. https://www.vernier.com/products/software/lp/ [Online; accessed 24-November-2017].

[21] Ž. Barba, L. Perpar, A. Ramšak, G. Simič, and L. Zadnik. Anizotropna vrv, 2011. http:// projlab.fmf.uni-lj.si/arhiv/2010_11/naloge/izdelki/anizotropna_vrv/meritve.html [Online; accessed 19-January-2018, in Slovenian]. 